\title{
Preserving Right Pre-motor and Posterior Prefrontal Cortices Contribute to Maintaining Overall Basic Emotion
}

\author{
Riho Nakajima ${ }^{1}$, Masashi Kinoshita ${ }^{2}$, Hirokazu Okita ${ }^{3}$, Zhanwen Liu $^{4}$ \\ and Mitsutoshi Nakada ${ }^{2 *}$
}

\begin{abstract}
'Department of Occupational Therapy, Faculty of Health Science, Institute of Medical, Pharmaceutical and Health Sciences, Kanazawa University, Kanazawa, Japan, ${ }^{2}$ Department of Neurosurgery, Faculty of Medicine, Institute of Medical, Pharmaceutical and Health Sciences, Kanazawa University, Kanazawa, Japan, ${ }^{3}$ Department of Physical Medicine and Rehabilitation, Kanazawa University Hospital, Kanazawa, Japan, ${ }^{4}$ Department of Neurosurgery, Graduate School of Medical Science, Kanazawa University, Kanazawa, Japan
\end{abstract}

\section{OPEN ACCESS}

Edited by:

Riki Matsumoto,

Kobe University, Japan

Reviewed by:

Jun Muto,

Fujita Health University, Japan Yoshiki Arakawa,

Kyoto University, Japan

*Correspondence:

Mitsutoshi Nakada

mnakada@med.kanazawa-u.ac.jp

Specialty section This article was submitted to Brain Imaging and Stimulation, a section of the journal Frontiers in Human Neuroscience

Received: 01 October 2020 Accepted: 25 January 2021 Published: 16 February 2021

Citation: Nakajima $R$, Kinoshita M, Okita $H$, Liu Z and Nakada M (2021) Preserving Right Pre-motor and Posterior Prefrontal Cortices Contribute to Maintaining Overall Basic Emotion. Front. Hum. Neurosci. 15:612890. doi: 10.3389/fnhum.2021.612890
Basic emotions such as happiness, sadness, and anger are universal, regardless of the human species, and are governed by specific brain regions. A recent report revealed that mentalizing, which is the ability to estimate other individuals' emotional states via facial expressions, can be preserved with the help of awake surgery. However, it is still questionable whether we can maintain the ability to understand others' emotions by preserving the positive mapping sites of intraoperative assessment. Here, we demonstrated the cortical regions related to basic emotions via awake surgery for patients with frontal glioma and investigated the usefulness of functional mapping in preserving basic emotion. Of the 56 consecutive patients with right cerebral hemispheric glioma who underwent awake surgery at our hospital, intraoperative assessment of basic emotion could be successfully performed in 22 patients with frontal glioma and were included in our study. During surgery, positive responses were found in 18 points in 12 patients (54.5\%). Of these, 15 points from 11 patients were found at the cortical level, mainly the premotor and posterior part of the prefrontal cortices. Then, we focused on cortical 15 positive mappings with 40 stimulations and investigated the types of emotions that showed errors by every stimulation. There was no specific rule for the regionemotional type, which was beyond our expectations. In the postoperative acute phase, the test score of basic emotion declined in nine patients, and of these, it decreased under the cut-off value $(Z$-score $\leq-1.65)$ in three patients. Although the total score declined significantly just after surgery $(p=0.022)$, it recovered within 3 months postoperatively. Our study revealed that through direct electrical stimulation (DES), the premotor and posterior parts of the prefrontal cortices are related to various kinds of basic emotion, but not a single one. When the region with a positive mapping site is preserved during operation, basic emotion function might be maintained although it declines transiently after surgery.

Keywords: basic emotion, awake surgery, intraoperative monitoring, glioma, right frontal lobe 


\section{INTRODUCTION}

Expression of emotion and meanings of facial expressions differ according to country or area. Basic emotion (e.g., anger, disgust, fear, happiness, sadness, and surprise) is a culturally universal expression and is governed by specific brain regions (Tracy and Randles, 2011). Happiness relates to the ventral prefrontal cortex, cingulate cortex, and ventral striatum, and sadness is governed by the medial prefrontal cortex, including the anterior cingulate cortex and middle frontal gyrus (MFG), while fear mainly involves the amygdala and insula (Vytal and Hamann, 2010; Celeghin et al., 2017; Gu et al., 2019). To support this knowledge, specific emotions are damaged by focal brain damage. For instance, it has been proven in humans and animals that the sense of fear is impaired by damage to the amygdala (Adolphs et al., 1994; Calder et al., 2001; Celeghin et al., 2017), and disgust might be affected by damage to the insula and basal ganglia (Calder et al., 2000, 2001; Suzuki et al., 2006). In contrast, it has also been argued that basic emotion is not necessarily governed by a specific brain region. That is, certain brain lesions relate not only to specific emotional states but also to several kinds of neurological/neuropsychological functions (Saarimäki et al., 2016; Celeghin et al., 2017). Specifically, the amygdala and medial-prefrontal cortex play a role in various emotions and are considered to be the key brain regions for basic emotion (Phan et al., 2002; Saarimäki et al., 2016; Huang et al., 2020). As for the laterality of basic emotion in healthy people, the bilateral cerebral hemispheres are involved (Vytal and Hamann, 2010; Saarimäki et al., 2016; Gu et al., 2019). However, it is considered that the laterality of emotional function is in the right cerebral hemisphere since emotional perception is damaged more severely in right lesions than in left lesions (KucharskaPietura et al., 2003; Yuvaraj et al., 2013; Suslow et al., 2016; Gainotti, 2019).

Previously, attempts to monitor emotional function during awake surgery for right cerebral hemispheric gliomas have been reported. Among them, Giussani et al. (2010) first reported intraoperative monitoring of emotional perception. They assessed six types of basic emotions, namely anger, happiness, fear, surprise, disgust, and sadness, in 18 patients with right cerebral hemispheric glioma. Positive mapping sites were found at five points in five cases, and they were superior to the middle temporal gyrus to the supramarginal gyrus. Later, Herbet et al. (2015) reported intraoperative monitoring of face-based mentalizing using the Reading the mind in the eyes (RME) test (Baron-Cohen et al., 2001). The research group demonstrated that the responsible region was found in the inferior frontal gyrus (IFG). Next, to verify previous data, the same research group investigated 27 patients with low-grade glioma and found positive mapping sites in the posterior IFG, dorsolateral prefrontal cortex, and posterior superior temporal gyrus at the cortical level (Yordanova et al., 2017). Their findings corresponded to those of previous neuroimaging studies (Adams et al., 2010; Celeghin et al., 2017).

Considering the above-mentioned background, the following two hypotheses arise: (1) Does direct electrical stimulation (DES) identify the region responsible for a single basic emotion?
(2) Can we preserve the ability to estimate another individual's emotional state by preserving positive mapping sites with focal DES? Here, we demonstrated the cortical regions related to basic emotions via awake surgery for patients with frontal glioma and investigated the usefulness of functional mapping for preserving basic emotion. Consequently, the premotor and posterior parts of the prefrontal cortices relate to various kinds of basic emotion, but not a single emotion. When the region is preserved with the help of awake brain mapping, it might recover within 3 months postoperatively, although it declines immediately after surgery. To the best of our knowledge, our study using DES is the first to demonstrate that the right premotor and posterior prefrontal cortices are related to various types of basic emotion.

\section{MATERIALS AND METHODS}

\section{Participants}

Of the 139 patients who underwent awake surgery at our hospital between August 2013 and December 2019, 56 patients had lesions were localized on the right side. Patients in whom preoperative magnetic resonance imaging (MRI) demonstrated that lesions were found in the temporal, occipital, and parietal lobes were excluded. Finally, 36 patients with right frontal glioma were matched to our inclusion criteria (Figure 1). We decided to perform awake surgery for all patients, considering age, infiltration region, and preoperative functional condition. Of these, 22 patients underwent intraoperative assessment of basic emotion successfully (mean age: 42.8 years). Patient details are shown in Table 1. Written informed consent was obtained from all patients. This study was performed according to the guidelines of the Internal Review Board of Kanazawa University and was approved by the Medical Ethics Committee of Kanazawa University (approval numbers 1797 and 2593).

\section{Awake Surgery}

Awake surgery was performed using the "asleep-awake-asleep" technique, where the cortical and subcortical brain mapping was performed using DES (Duffau et al., 2002). A bipolar electrode with 5-mm-spaced tips delivering a biphasic current (pulse frequency of $60 \mathrm{~Hz}$, pulse duration of $0.2 \mathrm{~ms}$, amplitude between 1.5 and $6 \mathrm{~mA}$, Nihon Kohden Neuromaster) was used in all cases. Patients were selected for functional monitoring during awake surgery, including visuospatial cognition, working memory, social cognition, basic emotion, and so on. In our patient group, 22 patients underwent monitoring for basic emotion. Although some reports have revealed the usefulness of functional mapping for emotional function (Herbet et al., 2015; Yordanova et al., 2017), intraoperative assessment for the neuropsychological function is still a new challenge in glioma surgery. Importantly, to achieve the best onco-functional balance for all glioma patients, we considered resection of the central part of the tumor, i.e., the enhanced lesion, and then performed intraoperative assessments on the extended resection.

To assess basic emotion intraoperatively, we used photographs of modified Japanese facial expressions of basic emotion series provided by Matsumoto and Ekman (1988). In the basic emotional test, a face of the eye region with two possible 


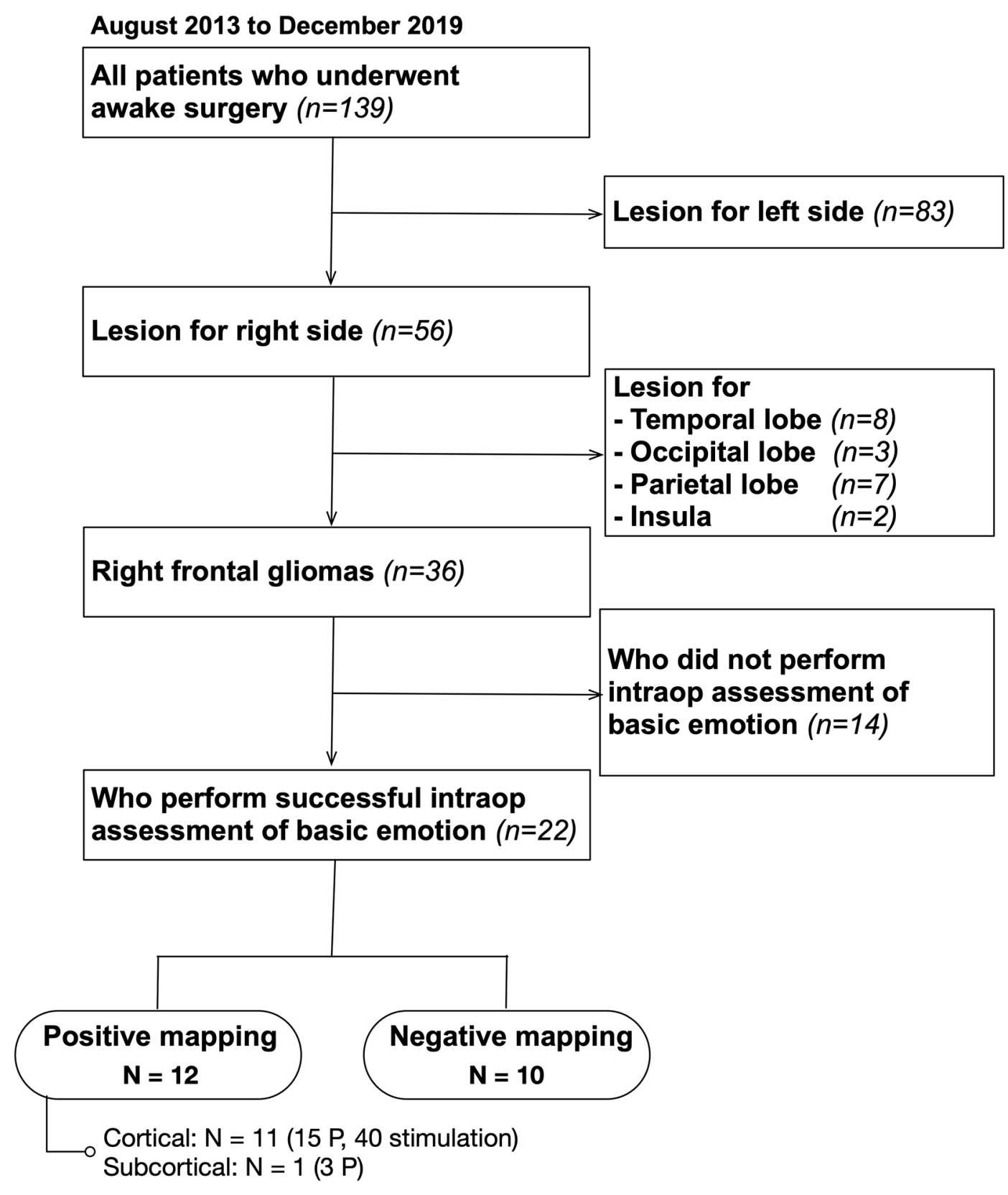

FIGURE 1 | Inclusion criteria. A total of 139 patients who underwent awake surgery in our institution were included; 36 of them matched our inclusion criteria. Of these, 22 patients underwent intraoperative monitoring of basic emotion successfully and were studied. P, point.

choices was presented on a computer monitor (Supplementary Figure 1). Patients were asked to select the most suitable emotional state for expressing the eyes from two choices within 2 s. Incorrect or delayed responses $(\geq 3 \mathrm{~s})$ were determined as positive responses. When positive responses were elicited in at least two out of three stimuli, the region was determined as a "positive response" and was preserved. Then, we overlaid each positive mapping site on each standardized MR image and studied the characteristics of incorrect responses during stimulation to positive mapping regions.

\section{Functional Assessment}

All patients underwent assessment of basic emotion over time until 3 months postoperatively. As for the assessment of basic emotion, we used the expression recognition test for adults, which is most widely used in Japan (Komatsu et al., 2012). It consists of 32 photographs of basic emotions, including happiness, sadness, anger, and surprise. For each of them, a photograph with five possible choices is presented, and patients are asked to select the most reasonable mental state from the choices. Of note, since there are racial 
TABLE 1 | Demographic and clinical characteristic of participants.

\begin{tabular}{lc}
\hline Factors & Value \\
\hline Age & $42.8 \pm 15.6(14-72)$ \\
Sex; male/female & $10 / 12$ \\
Handedness; Right/left & $20 / 2$ \\
WHO grade & I 2, II 7, III 9, IV 4 \\
Histology & \\
$\quad$ Dysembryoplastic neuroepithelial tumor & 2 \\
Diffuse astrocytoma & 2 \\
Oligodendroglioma & 4 \\
Ependymoma & 1 \\
Anaplastic astrocytoma & 2 \\
Anaplastic oligodendroglioma & 7 \\
Glioblastoma & 4 \\
IDH-1; mutant/wild type/ND & $18 / 3 / 1$ \\
1p19q; codeletion/intact/ND & $10 / 10 / 2$ \\
Preop MMSE score & $28.3 \pm 2.0$ \\
\hline
\end{tabular}

MMSE, mini-mental state examination; ND, not detected.

differences, assessment mainly prepared for Europeans and North Americans, such as the RME test, is not suitable for Asians (Baron-Cohen et al., 2001).

\section{Magnetic Resonance Imaging and Lesion Mapping}

Structural MRI was performed during a 3-month postoperative period as the standard care. The images were acquired using a conventional high-resolution three-dimensional (3D) T1-weighted 3.0 T MRI scanner (Signa ${ }^{\mathrm{TM}}$ Excite HDx 3.0T; GE Healthcare, Little Chalfont, UK). Each MR image was reconstructed on the Montreal Neurological Institute (MNI) template using SPM12 software ${ }^{1}$, which runs in MATLAB ${ }^{2}$. We composed the resection cavities manually using the MRIcron software $^{3}$. Each step was performed initially by RN and then systematically checked by a neurosurgeon (MK).

\section{Spatial Topography of Stimulations}

The spatial topography of the stimulation points for emotion recognition was plotted in the following steps. First, the positive mapping sites were plotted on the corresponding original 3DT1-images for each patient using iPlan Stereotaxy 3.0 software (BrainLab), according to operative reports and intraoperative video records. The exact locations of the DES points were determined by considering their spatial relationships to various anatomical landmarks (the gyri, sulci, vessel, and midline), as previously described (Tate et al., 2014; Nakajima et al., 2020). Next, each positive mapping site of emotion recognition on the original MR images was transferred to normalized T1-images. Then, the spatial location of the positive mappings was overlaid on the 3D MNI template using the MRIcroGL software ${ }^{4}$. Finally, the anatomical location of positive mapping sites on the $3 \mathrm{D}$ MNI template was checked again in comparison to the original operative records. Each step was first undertaken by the $\mathrm{RN}$ and then systematically checked by a neurosurgeon (MK). For

\footnotetext{
${ }^{1}$ http://www.fil.ion.ucl.ac.uk/spm/software/spm12/

${ }^{2} \mathrm{http}: / /$ www.mathworks.com/products/matlab/

${ }^{3}$ http://www.mccauslandcenter.sc.edu/mricro/mricron/

${ }^{4}$ http://www.cabiatl.com/mricrogl/
}

all positive mapping sites, we counted the times of stimulation for the same point (maximum, three) via intraoperative video recording. We then examined the types of emotions that were errors for each DES point. Besides, stimulated sites with negative responses were also determined using intraoperative multiscreen video record as the same procedure of positive mapping sites above mentioned. They were all overlapped on the $3 \mathrm{D}$ MNI template.

\section{Statistical Analysis}

First, all row scores of the expression recognition test and other neuropsychological assessments were converted to $Z$-scores using the mean and standard deviations of the age-matched controls. To compare the accuracy of basic emotion recognition over time, the Steel-Dwass analysis was used. The non-parametric test was used in the current study because our patients' data did not follow a normal distribution. All statistical analyses were performed using statistical analysis software (JMP, version 14.3.0; SAS Institute, Incorporation).

\section{RESULTS}

\section{Anatomical Data}

The overlap map of resection cavities that perform an intraoperative assessment of basic emotion $(n=22)$ demonstrated that the greatest overlap was in the posterior deep white matter of the medial frontal lobe (Figure 2).

\section{Intraoperative Findings}

Positive responses were found in 18 points in 12 cases $(54.5 \%)$. Of these, 15 positive mappings from 11 patients were found at the cortical level, mainly the premotor and posterior part of the prefrontal cortices; with 3, 5, and 7 points for the superior frontal gyrus (SFG), MFG, and IFG, respectively (Figure 3Aa). Stimulated regions with negative responses of these patients are shown in Figure $\mathbf{3 A b}$. Then, we focused on patients whose positive mappings for basic emotion were identified during surgery in the following analysis. To note that three positive mapping sites from one patient of subcortical level, which were in the deep part of the MFG and IFG, were not investigated further in this study.

We found 56 total stimulations of positive mappings at the cortical level. Incorrect responses per total number of stimulations were 10 out of 14,11 out of 15 , and 19 out of 27 times, in the SFG, MFG, and IFG, respectively (Figure 3B). Details of patients' incorrect responses were summarized in Supplementary Table 1 . We investigated the relationship between the spatial location of incorrect responses and the emotional type and found that incorrect responses for happiness (red circle) were found only in the IFG, and errors for neutral faces (green circles) were found only in the SFG and MFG, but not in the IFG (Figures 3B, 4). In contrast, errors for sadness were found in the MFG and IFG, but not in the SFG. Also, incorrect responses were found in almost all kinds of emotions in each gyrus, and it had no other specific rule for regionemotional type. 


\section{Lateral view}

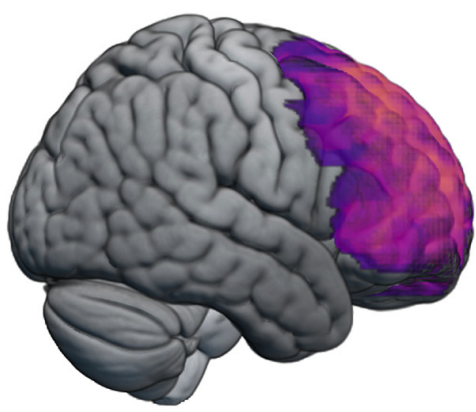

Frontal view

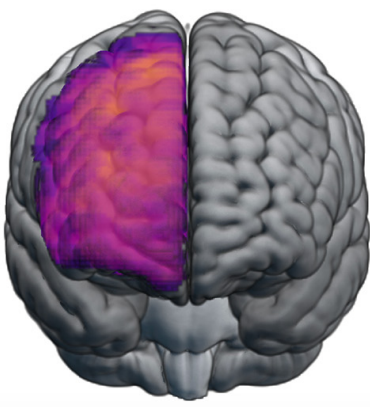

\section{Medial view}

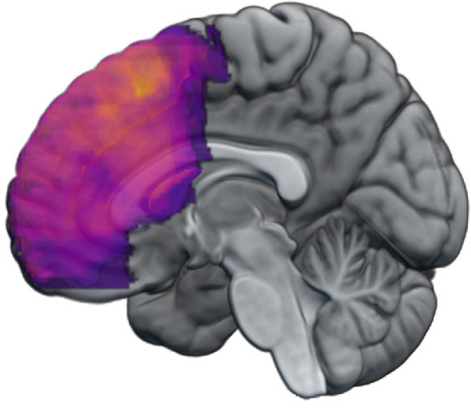

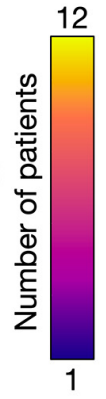
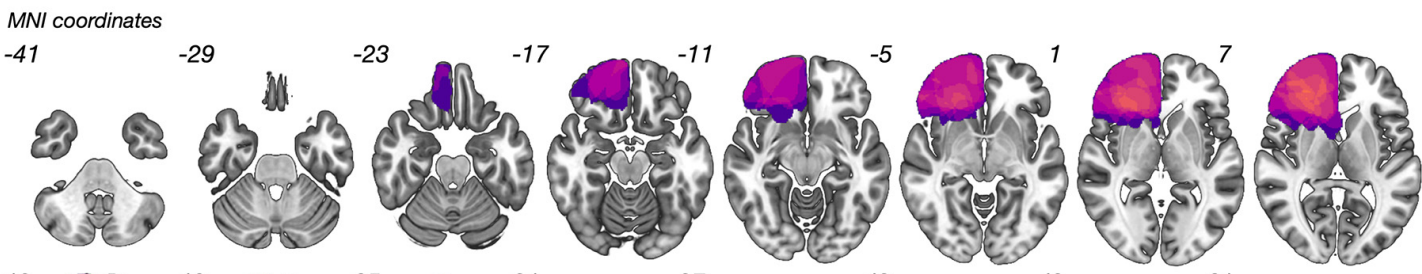

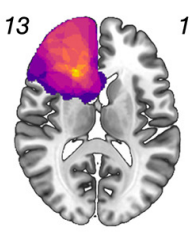

Right
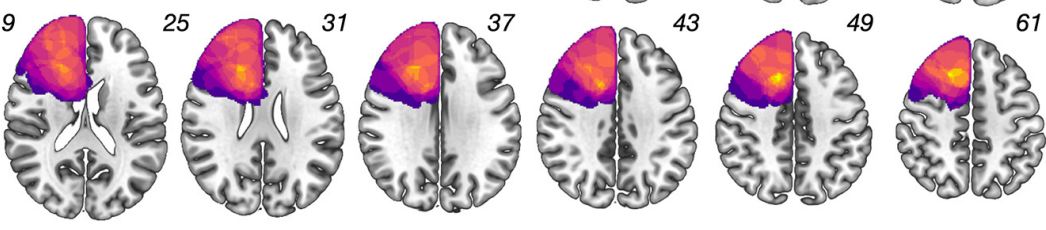

61

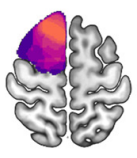

$\mathrm{N}=22$

Left

FIGURE 2 | Overlap map of resection cavities showing that the posteromedial prefrontal cortex and its deep part had the greatest overlap. Numbers at the upper left of the slice images indicate the coordinates of the Montreal Neurological Institute (MNI) template.

After surgery, the basic emotion test score declined in nine patients compared with the preoperative score, and of these, it declined under the cut-off value $(Z \leq-1.65)$ in three patients (Figure 5A, details are shown in Supplementary Table 2). Although the total score declined significantly just after surgery (preoperative, $0.21 \pm 0.61$; postoperative 1 week, $-0.98 \pm 1.15$; $p=0.022$ ), it recovered to the same preoperative level within 3 months postoperatively $(-0.28 \pm 0.78$; Figure $5 B)$. In our patient group, there was no postoperative significant decline of neuropsychological function such as attention or executive function which may influence the accuracy of basic emotion (Supplementary Figure 2).

\section{Illustrative Cases}

Two illustrative cases are shown in Figure 6. The patient in case 1 was a 62-year-old man, whose tumor (glioblastoma, IDH-mutant) extended in the posterior pre-motor to the prefrontal area. Preoperatively, the results of neuropsychological examination revealed that attention and executive functions declined, while basic emotion was normal $(Z=-0.41)$. During surgery, incorrect responses for disgust, fear, anger, and sadness were found at the posterior part of the MFG. Because incorrect responses were reproducible, the point was determined as positive mapping and it was considered to be the limit of resection. Postoperative examinations revealed that the extent of resection was $100 \%$, and his test score for basic emotion declined under the cut-off value $(Z=-1.79)$ but recovered within 3 months postoperatively. The patient in case 2 was a 39-year-old woman, whose tumor (anaplastic astrocytoma, IDH-wild type) infiltrated in the pre-motor to the prefrontal areas, and decided to undergo a second surgery. Preoperatively, a neuropsychological examination was performed, and her brain function was almost intact, except for a slight decline in working memory. She underwent awake surgery with the monitoring of basic emotion. Incorrect responses for fear, contempt, and disgust induced by DES were found at the posterior part of the IFG. Since the point was considered to be positive mapping, it was determined as the limit of resection. Postoperative MR images revealed that extended resection was performed. Postoperatively, her emotional function did not decline.

\section{DISCUSSION}

The primary aim of the current study was to reveal the cortical brain region related to basic emotion, and the secondary aim was to investigate the usefulness of functional mapping during awake surgery for preserving basic emotion. Consequently, the 


\section{A Spatial location of the positive mapping sites}
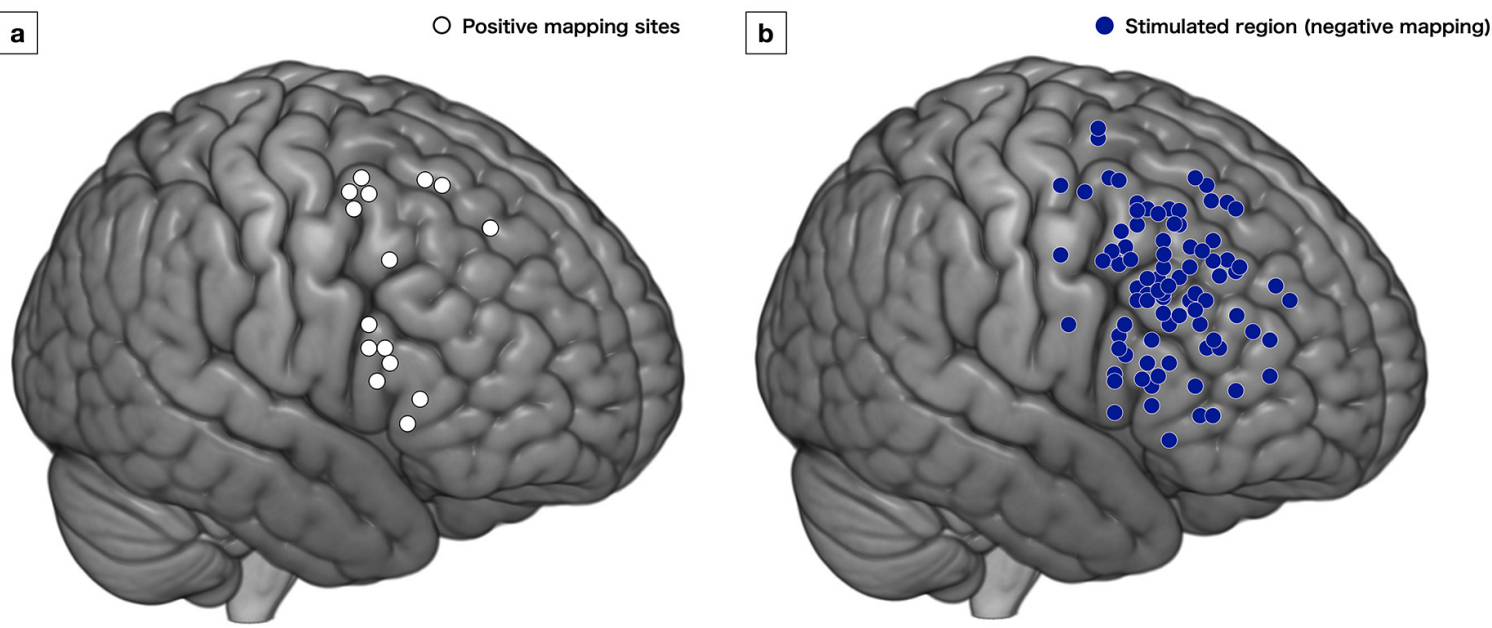

\section{B Type of error for each positive mapping}

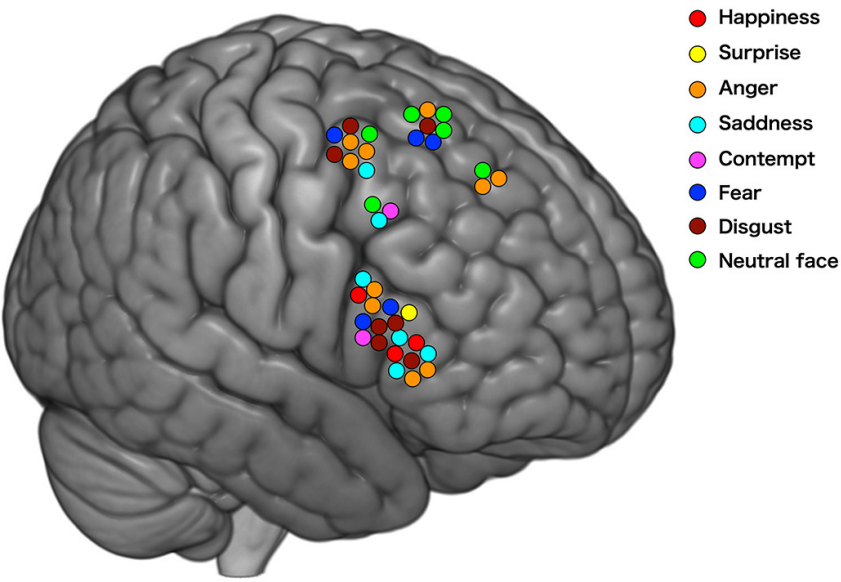

FIGURE 3 | Distribution of the positive mapping sites (Aa, white circle) and regions with negative responses (Ab, dark blue circle) at the cortical level. Each positive mapping site is stimulated two or three times, and when the error or delayed responses are observed at least twice, the region is considered as "positive." The lower image (B) shows the type of errors for each positive mapping. The type of emotion for which the incorrect responses were elicited is shown. Red, happiness; yellow, surprise; orange, anger; cyan, sadness; pink, contempt; blue, fear; brown, disgust; light green, neutral face.

premotor and posterior parts of the prefrontal cortices are related to various kinds of basic emotion, but no frontal region is related to a single emotion. When monitoring of basic emotion is conducted during surgery and the identified localization is preserved successfully, even though the accuracy of basic emotion might be decreased temporally, it might recover. Our study is the first to demonstrate that the right premotor and posterior prefrontal cortices are related to all kinds of basic emotion, rather than a single type.

\section{Brain Region Relating to Basic Emotion}

Through DES during awake surgery, we found that the premotor and posterior prefrontal cortices are related to various kinds of basic emotion, but we did not detect differences depending on emotional types. Previously, several studies have revealed the involvement of the premotor and prefrontal cortices in basic emotion. Indeed, the prefrontal cortex is known to be related to several kinds of emotions, such as happiness, sadness, anger, disgust, and fear (Vytal and Hamann, 2010; Dal Monte et al., 2013; Celeghin et al., 2017; Zhao et al., 2017; Gu et al., 2019). Specifically, an fMRI study demonstrated that the MFG was the common region for various kinds of facial recognition of basic emotion (Diano et al., 2017). Also, another study found that the gray matter volume of the IFG was positively related to the score of facial expression recognition for overall basic emotion (Uono et al., 2017). The mirror neuron system is also known to relate to face-based emotion recognition (Herbet et al., 2014; Perry et al., 2017). A mirror neuron is a neuron that acts selectively when observing another's action, and it senses automatically another's emotional state from facial 


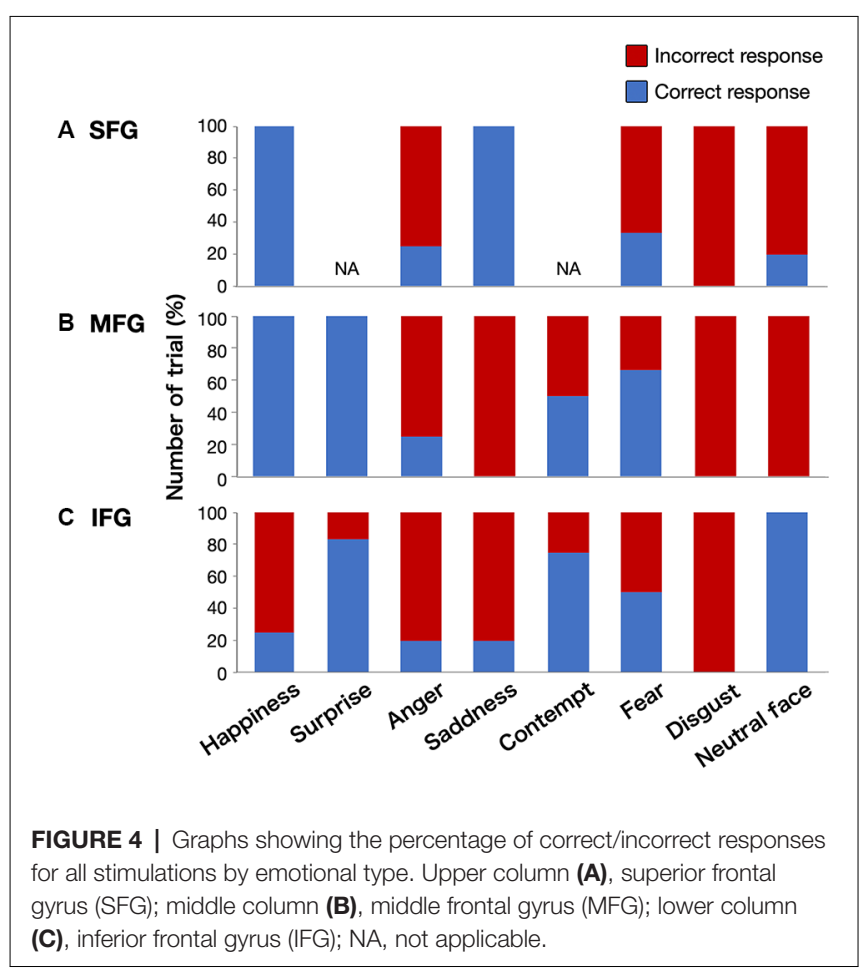

expression or bodily action (Moor et al., 2012). Mirror neuron exists mainly in the premotor cortex, inferior parietal lobe, posterior superior temporal sulcus, anterior cingulate cortex, amygdala, and anterior insula (Rizzolatti et al., 1996; Lacoboni and Dapretto, 2006; Cattaneo and Rizzolatti, 2009). Among them, the premotor cortex, as well as insula considered as a core neural system for emotional processing (Jeon and Lee, 2018). Collectively, the frontal lobe is considered a key region for basic emotion (Saarimäki et al., 2016). That is, these results also indicated that the specific frontal region is not related to a single emotion (Kucharska-Pietura et al., 2003; Vytal and Hamann, 2010). Moreover, the prefrontal cortex, especially the ventral prefrontal cortex is structurally and functionally connected with the amygdala which is a center of various emotions ( $\mathrm{Li}$ et al., 2013; Morawetz et al., 2017). The prefrontal-amygdala connectivity relates to emotion in healthy people (Clewett et al., 2014; Fernandes et al., 2019; Ibrahim et al., 2019). These findings suggest that premotor and prefrontal cortices play a role in basic emotion.

Looking in detail, the scores of sadness and disgust tend to be worse than other emotions. It is probably because of cultural differences between Caucasian-Americans and native Japanese for recognition of negative emotion (Harada et al., 2020). The Japanese are weak in expression recognition for negative emotion including sadness and disgust compared with Americans (Shioiri et al., 1999).

The positive mapping sites obtained in the current study mostly corresponded to previously reported results of intraoperative assessment of face-based mentalizing (Herbet et al., 2015; Yordanova et al., 2017). Interestingly, our study used basic emotion as an intraoperative task, while the study reported by Herbet et al. (2015) and Yordanova et al. (2017) used the RME-test, requiring patients to discriminate a more complex mental state of the face than a basic emotion. Several neuroimaging studies have reported the involvement of the right premotor and prefrontal cortices to the RME-test by several neuroimaging studies (Castelli et al., 2010; Herbet et al., 2014; Domínguez et al., 2019). This means that the premotor and posterior part of the prefrontal cortices relates to general face-based emotional perception, rather than a specific type of emotion. Taken together, assessment for all types of the emotional state rather than specific emotion might be appropriate for intraoperative monitoring at the right premotor and prefrontal regions. In contrast, a previous report revealed that the DES for the anterior insula induced response of a particular emotional type, such as anger and sadness (Motomura et al., 2019). We can take into consideration to use specific emotional type as an intraoperative assessment for the regions which induce unique emotional deficit by the DES.

\section{The Usefulness of DES for Preserving Basic Emotion}

In the current study, basic emotion could be maintained by preserving regions with positive mappings during surgery, even though it declined immediately after surgery. This finding corresponds to that of previous studies that performed intraoperative awake mapping of emotional function (Giussani et al., 2010; Papagno et al., 2016). Additionally, the postoperative course is similar to that of several kinds of neuropsychological/neurological function assessed during awake surgery (De Witt Hamer et al., 2012; Nakajima et al., 2019).

Ideally, it is necessary to compare functional outcomes between the with- and without-intraoperative evaluation groups to determine the usefulness of awake mapping of basic emotion. However, this method is impossible because of the following reasons. First, setting a control group that does not undergo intraoperative mapping is difficult from an ethical point of view. Since, we know the advantages of intraoperative monitoring in awake conditions for several neuropsychological functions, they are still challenging (Giussani et al., 2010; Herbet et al., 2015; Papagno et al., 2016; Yordanova et al., 2017). Also, although there were no significant differences in the mean score of basic emotion between the evaluation group and non-evaluation group before surgery (Supplementary Table 3), the score of the latter declined slightly in 6 out of 14 patients (42.9\%) preoperatively ( $Z \leq-1.65$, data not shown). Therefore, we decided not to use intraoperative assessment for some patients. Hence, we could not simply compare functional outcomes between the evaluation and non-evaluation groups.

Recently, the emotional function has gradually gained more attention in brain tumor surgery (Pertz et al., 2020). For instance, a report suggested that there is a high possibility of social cognition including the emotional function of patients with brain tumors being damaged preoperatively (Goebel et al., 2018). However, even now, the postoperative decline in emotional function in the right cerebral hemispheric glioma tends to be considered to recover 


\section{A Time series score of individuals}

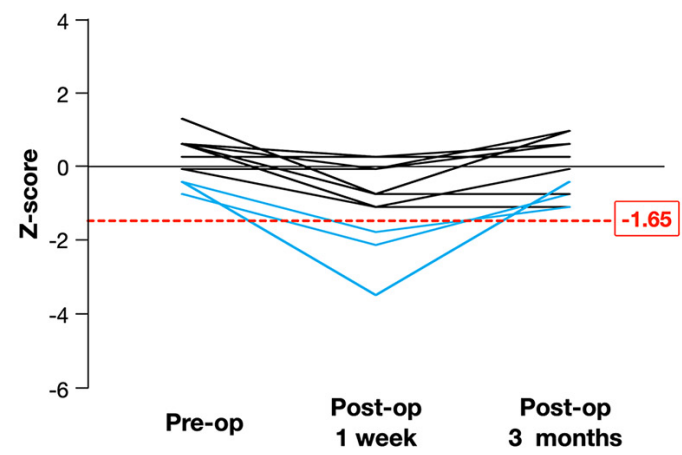

\section{B Comparison of test score between times}

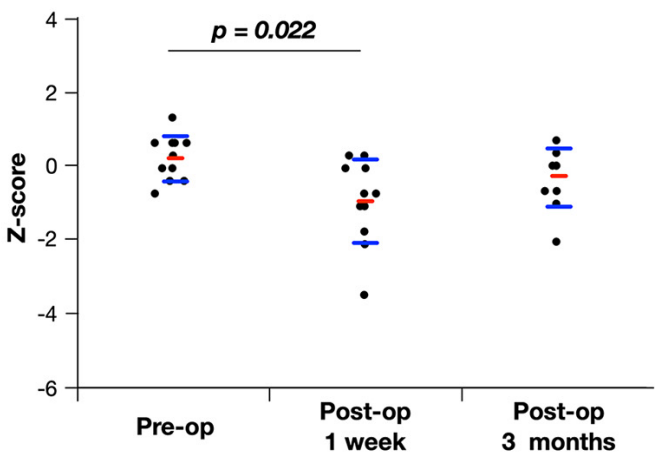

FIGURE 5 | Time course of assessment for basic emotion in patients whose positive mappings were identified during awake surgery. The figure of time series of individuals $(\mathbf{A})$ indicates that three patients declined under cut-off value $(Z \leq-1.65)$ just after surgery, but they recovered completely. When scores are compared among time series using Steel-Dwass analysis (B), though the mean value declined significantly just after surgery, there is no decline at 3 months postoperatively compared with the preoperative score.

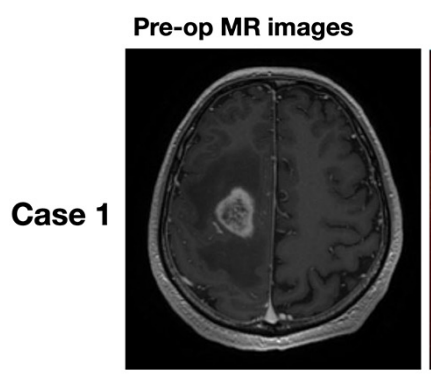

Intra-op findings
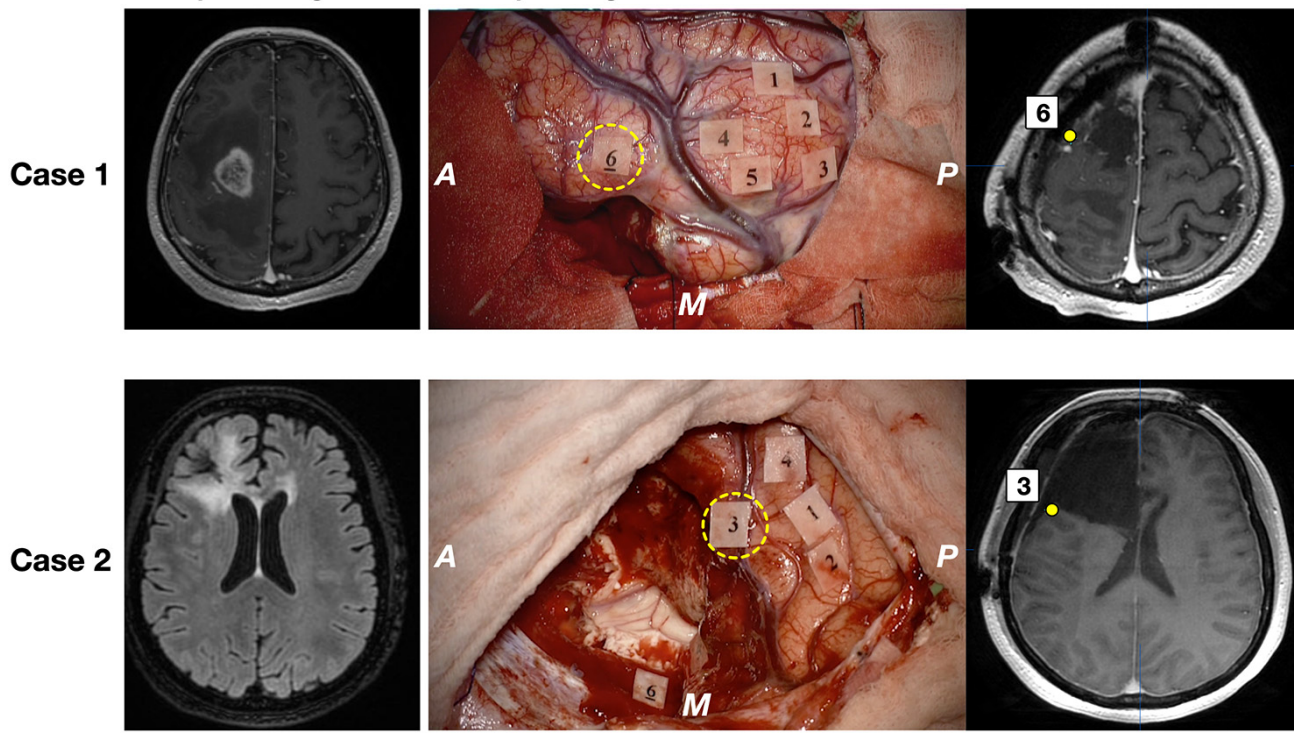

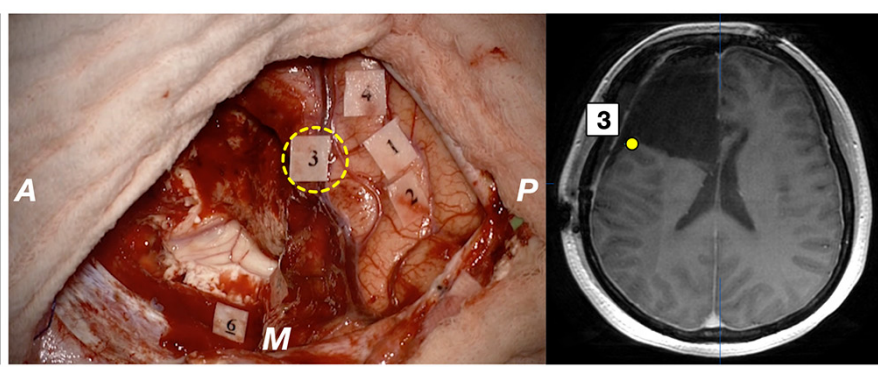

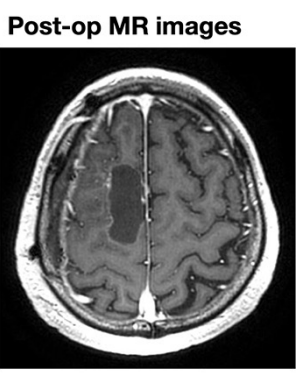

$\mathrm{GD}+\mathrm{T} 1$

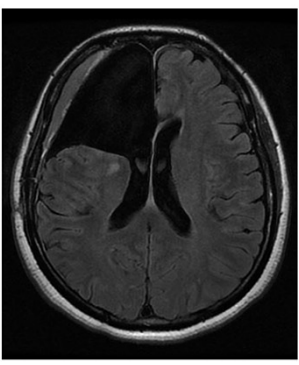

FLAIR

FIGURE 6 | Pre- and postoperative magnetic resonance (MR) images (gadolinium-enhanced T1 image for Case 1 and FLAIR image for Case 2) and intraoperative findings of illustrative two cases are shown. MR images and number tags with the yellow circle of the middle column show the exact location of positive mappings of basic emotion identified using iPlan Stereotaxy 3.0 software (BrainLab). A: anterior; P: posterior; M: medial side; yellow broken circle, tags of incorrect responses for basic emotion. Tags and symptoms elicited by electrical stimulation are shown. Case 1: Tag 1-5, involuntary movement; Tag 6, emotion recognition; Case 2: Tag 1 and 2, dysarthria; Tag 3, emotion recognition; Tag 4, negative motor response; Tag 6, Stroop test.

completely in a relatively short period following surgery (Campanella et al., 2015; Mattavelli et al., 2019; Pertz et al., 2020).

In contrast, there is an important study attracting clinicians' attention, although the number of cases is insufficient (Herbet et al., 2013). The authors investigated the postoperative course of emotion recognition in 10 patients with low-grade glioma divided into two groups according to resected lesion; those with the resected right dorsomedial prefrontal cortex and those with resected right IFG. As a result, although postoperative emotion recognition deficits in the former group were temporary, those in the latter group did not recover beyond 3 months postoperatively. Another study revealed that overall emotion recognition accuracy at a chronic phase in patients with the resected prefrontal cortex, especially the orbitofrontal cortex and medial prefrontal cortex, was significantly declined in comparison to the control group 
(Jenkins et al., 2014). These previous findings suggest that damage to a specific region of the frontal lobe may lead to an irreversible deficit in emotion recognition. Because the basic emotion of our patient group was maintained, in patients whose localization of basic emotion could be identified during surgery, intraoperative monitoring of basic emotion should be considered in glioma surgery for the right frontal lobe.

\section{Limitations}

There are several limitations to the current study. First, as mentioned in the preceding section, we cannot validate the usefulness of intraoperative monitoring of basic emotion compared to the control group. Although several previous reports have revealed the utility of intraoperative monitoring for right cerebral hemispheric function including emotional function, assessment of right frontal lobe function is still a challenge (Thiebaut de Schotten et al., 2005; Herbet et al., 2015, 2016; Kinoshita et al., 2016; Nakajima et al., 2018). The usefulness of intraoperative monitoring of neuropsychological function should be considered from various aspects, such as the extent of resection, progression-free survival, overall survival, functional outcome, and postoperative social lives of patients. Another limitation is the small number of cases. However, the number of cases in previous studies on intraoperative monitoring of basic emotion was also not many; there were 5 of 18 patients with right cerebral hemispheric gliomas in Giussani et al. (2010), 13 patients with left insula glioma in Papagno et al. (2016), and 11 patients with right insula glioma who complete the intraoperative task in Motomura et al. (2019). Our study investigated the usefulness of intraoperative monitoring for basic emotion with a similar or marginally higher number of patients than the previous reports, with a focus on the right frontal lobe. Our patient's group includes both low-grade and high-grade gliomas, although they are not biologically the same. However, several recent studies that investigated functional localization via awake brain mapping include both tumor types and obtain meaningful results (GrasCombe et al., 2012; Saito et al., 2016; Nakajima et al., 2018; Puglisi et al., 2018; Rolland et al., 2018; Nakada et al., 2020). Additionally, we could not study the white matter tract, since we found only a few positive mapping sites at the subcortical level in our patient group. Third, the current study is a retrospective study of intraoperative monitoring in a clinical setting. Hence, the cortical area that can be assessed is limited to the surrounding brain tumor within the operative field. Moreover, it is unclear whether the same results can be obtained in the normal brain since slow-growing gliomas might lead to reorganization with the functional shift from original localization at the cortical level (Duffau, 2014). Although cortical reorganization for language and motor function in gliomas has been reported (Saito et al., 2016; Southwell et al., 2016; Nakajima et al., 2020), brain plasticity regarding higher brain function, such as basic emotion, is poorly understood. In support of our results, some previous reports revealed that the premotor and posterior parts of the prefrontal cortices are related to basic emotion in the normal brain (Diano et al.,
2017; Uono et al., 2017). These reports showed a relatively smaller area for emotion recognition than our results. Our results might show a broader region than the original functional localization, since our data may include the region with a functional shift.

Finally, to date, the concept for basic emotion itself and localization for each emotional type is a matter of debate without consensus (Eugène et al., 2003; Touroutoglou et al., 2015; Hutto et al., 2018; Keltner et al., 2019). Further study will be required with a large number of cases to reveal which emotional states should be used in certain brain regions for intraoperative mapping, and its method for suitable assessment.

\section{CONCLUSION}

The premotor and posterior parts of the prefrontal cortices are related to the different types of basic emotion. When the region with a positive mapping site is preserved, it might recover within 3 months postoperatively even though it declines immediately after surgery. Our findings will be useful in the field of neuroscience as well as for clinicians who are engaging in the treatment of brain damage, such as neurosurgery/neurology and rehabilitation.

\section{DATA AVAILABILITY STATEMENT}

The original contributions presented in the study are included in the article/Supplementary Material, further inquiries can be directed to the corresponding author.

\section{ETHICS STATEMENT}

This study was performed according to the guidelines of the Internal Review Board of Kanazawa University and was approved by the Medical Ethics Committee of Kanazawa University (approval numbers 1797 and 2593). The patients/participants provided their written informed consent to participate in this study. Written informed consent was obtained from the individual(s) for the publication of any potentially identifiable images or data included in this article.

\section{AUTHOR CONTRIBUTIONS}

$\mathrm{MN}$ and $\mathrm{RN}$ : conception and design, drafting article. $\mathrm{RN}$, $\mathrm{MK}$, and $\mathrm{HO}$ : acquisition of data. RN, MK, and ZL: analysis and interpretation of data. MN: study supervision. All authors contributed to the article and approved the submitted version.

\section{FUNDING}

This work was supported by Japan Society for the Promotion of Science (JSPS) KAKENHI Grant Numbers 18H03126, 18K19606, 19K22801, 20K21649, and 20KK0206. 


\section{SUPPLEMENTARY MATERIAL}

The Supplementary Material for this article can be found online at: https://www.frontiersin.org/articles/10.3389/fnhum. 2021.612890/full\#supplementary-material.

SUPPLEMENTARY FIGURE 1 | Schema of intraoperative assessment for basic emotion.

SUPPLEMENTARY FIGURE 2 | These images show results of neuropsychological assessments which may influence the accuracy of emotion

\section{REFERENCES}

Adams, R. B. Jr., Rule, N. O., Franklin, R. G. Jr., Wang, E., Stevenson, M. T., Yoshikawa, S., et al. (2010). Cross-cultural reading the mind in the eyes: an fMRI investigation. J. Cogn. Neurosci. 22, 97-108. doi: 10.1162/jocn.2009. 21187

Adolphs, R., Tranel, D., Damasio, H., and Damasio, A. (1994). Impaired recognition of emotion in facial expressions following bilateral damage to the human amygdala. Nature 372, 669-672. doi: 10.1038/37 $2669 \mathrm{a} 0$

Baron-Cohen, S., Wheelwright, S., Hill, J., Raste, Y., and Plumb, I. (2001). The "Reading the Mind in the Eyes" Test revised version: a study with normal adults and adults with Asperger syndrome or high-functioning autism. J. Child Psychol. Psychiatry 42, 241-251. doi: 10.1111/1469-7610. 00715

Calder, A. J., Keane, J., Manes, F., Antoun, N., and Young, A. W. (2000). Impaired recognition and experience of disgust following brain injury. Nat. Neurosci. 3, 1077-1078. doi: 10.1038/80586

Calder, A. J., Lawrence, A. D., and Young, A. W. (2001). Neuropsychology of fear and loathing. Nat. Rev. Neurosci. 2, 352-363. doi: 10.1038/350 72584

Campanella, F., Fabbro, F., Ius, T., Shallice, T., and Skrap, M. (2015). Acute effects of surgery on emotion and personality of brain tumor patients: surgery impact, histological aspects, and recovery. Neuro Oncol. 17, 1121-1131. doi: 10.1093/neuonc/nov065

Castelli, I., Baglio, F., Blasi, V., Alberoni, M., Falini, A., Liverta-Sempio, O., et al. (2010). Effects of aging on mindreading ability through the eyes: an fMRI study. Neuropsychologia 48, 2586-2594. doi: 10.1016/j.neuropsychologia.2010. 05.005

Cattaneo, L., and Rizzolatti, G. (2009). The mirror neuron system. Arch. Neurol. 66, 557-560. doi: 10.1001/archneurol.2009.41

Celeghin, A., Diano, M., Bagnis, A., Viola, M., and Tamietto, M. (2017). Basic emotions in human neuroscience: neuroimaging and beyond. Front. Psychol. 8:1432. doi: 10.3389/fpsyg.2017.01432

Clewett, D., Bachman, S., and Mather, M. (2014). Age-related reduced prefrontal-amygdala structural connectivity is associated with lower trait anxiety. Neuropsychology 28, 631-642. doi: 10.1037/neu00 00060

Dal Monte, O., Krueger, F., Solomon, J. M., Schintu, S., Knutson, K. M., Strenziok, M., et al. (2013). A voxel-based lesion study on facial emotion recognition after penetrating brain injury. Soc. Cogn. Affect. Neurosci. 8, 632-639. doi: 10.1093/scan/nss041

De Witt Hamer, P. C., Robles, S. G., Zwinderman, A. H., Duffau, H., and Berger, M. S. (2012). Impact of intraoperative stimulation brain mapping on glioma surgery outcome: a meta-analysis. J. Clin. Oncol. 30, 2559-2565. doi: 10.1200/JCO.2011.38.4818

Diano, M., Tamietto, M., Celeghin, A., Weiskrantz, L., Tatu, M. K., Bagnis, A., et al. (2017). Dynamic changes in amygdala psychophysiological connectivity reveal distinct neural networks for facial expressions of basic emotions. Sci. Rep. 7:45260. doi: 10.1038/srep45260

Domínguez, D. J., Nott, Z., Horne, K., Prangley, T., Adams, A. G., Henry, J. D., et al. (2019). Structural and functional brain correlates of theory of mind impairment post-stroke. Cortex 121, 427-442. doi: 10.1016/j.cortex.2019. 09.017

Duffau, H. (2014). Diffuse low-grade gliomas and neuroplasticity. Diagn. Intervent. Imaging 95, 945-955. doi: 10.1016/j.diii.2014.08.001 recognition; (A) Mini-mental state examination for general cognitive function; (B) letter cancellation test (\%-correct) for selective attention; (C) letter cancellation test (time required) for processing speed; (D) Stroop test for attentional control or executive function. There were no significant differences through the course using Steel-Dwass analysis.

SUPPLEMENTARY TABLE 1 | Details of patients' incorrect responses.

SUPPLEMENTARY TABLE 2 | Details of time series score of individuals.

SUPPLEMENTARY TABLE 3 | Demographic and clinical factor for each group.

Duffau, H., Capelle, L., Sichez, N., Denvil, D., Lopes, M., Sichez, J. P., et al. (2002). Intraoperative mapping of the subcortical language pathways using direct stimulations. An anatomo-functional study. Brain 125, 199-214. doi: 10.1093/brain/awf016

Eugène, F., Lévesque, J., Mensour, B., Leroux, J. M., Beaudoin, G., Bourgouin, P., et al. (2003). The impact of individual differences on the neural circuitry underlying sadness. NeuroImage 19, 354-364. doi: 10.1016/s10538119(03)00121-6

Fernandes, H. M., Cabral, J., van Hartevelt, T. J., Lord, L. D., Gleesborg, C., Moller, A., et al. (2019). Disrupted brain structural connectivity in pediatric bipolar disorder with psychosis. Sci. Rep. 9:13638. doi: 10.1038/s41598-01950093-4

Gainotti, G. (2019). A historical review of investigations on laterality of emotions in the human brain. J. Hist. Neurosci. 28, 23-41. doi: 10.1080/0964704X.2018. 1524683

Giussani, C., Pirillo, D., and Roux, F. E. (2010). Mirror of the soul: a cortical stimulation study on recognition of facial emotions. J. Neurosurg. 112, 520-527. doi: 10.3171/2009.5.JNS081522

Goebel, S., Mehdorn, H. M., and Wiesner, C. D. (2018). Social cognition in patients with intracranial tumors: do we forget something in the routine neuropsychological examination? J. Neurooncol. 140, 687-696. doi: $10.1007 / \mathrm{s} 11060-018-3000-8$

Gras-Combe, G., Moritz-Gasser, S., Herbet, G., and Duffau, H. (2012). Intraoperative subcortical electrical mapping of optic radiations in awake surgery for glioma involving visual pathways. J. Neurosurg. 117, 466-473. doi: $10.3171 / 2012.6$.JNS111981

Gu, S., Wang, F., Cao, C., Wu, E., Tang, Y. Y., and Huang, J. H. (2019). An integrative way for studying neural basis of basic emotions with fMRI. Front. Neurosci. 13:628. doi: 10.3389/fnins.2019.00628

Harada, T., Mano, Y., Komeda, H., Hechtman, L. A., Pornpattananangkul, N., Parrish, T. B., et al. (2020). Cultural influences on neural systems of intergroup emotion perception: an fMRI study. Neuropsychologia 137:107254. doi: 10.1016/j.neuropsychologia.2019.107254

Herbet, G., Lafargue, G., Bonnetblanc, F., Moritz-Gasser, S., and Duffau, H. (2013). Is the right frontal cortex really crucial in the mentalizing network? A longitudinal study in patients with a slow-growing lesion. Cortex 49 , 2711-2727. doi: 10.1016/j.cortex.2013.08.003

Herbet, G., Lafargue, G., Bonnetblanc, F., Moritz-Gasser, S., Menjot de Champfleur, N., and Duffau, H. (2014). Inferring a dual-stream model of mentalizing from associative white matter fibres disconnection. Brain 137, 944-959. doi: 10.1093/brain/awt370

Herbet, G., Lafargue, G., Moritz-Gasser, S., Bonnetblanc, F., and Duffau, H. (2015). Interfering with the neural activity of mirror-related frontal areas impairs mentalistic inferences. Brain Struct. Funct. 220, 2159-2169. doi: 10.1007/s00429-014-0777-x

Herbet, G., Moritz-Gasser, S., and Duffau, H. (2016). Direct evidence for the contributive role of the right inferior fronto-occipital fasciculus in non-verbal semantic cognition. Brain Struct. Funct. 222, 1597-1610. doi: 10.1007/s00429016-1294-X

Huang, Y.-A., Dupont, P., Van de Vliet, L., Jastorff, J., Peeters, R., Theys, T., et al. (2020). Network level characteristics in the emotion recognition network after unilateral temporal lobe surgery. Eur. J. Neurosci. 52, 3470-3484 doi: 10.1111/ejn.14849

Hutto, D. D., Robertson, I., and Kirchhoff, M. D. (2018). A new, better BET: rescuing and revising basic emotion theory. Front. Psychol. 9:1217. doi: 10.3389/fpsyg.2018.01217 
Ibrahim, K., Eilbott, J. A., Ventola, P., He, G., Pelphrey, K. A., McCarthy, G., et al. (2019). Reduced amygdala-prefrontal functional connectivity in children with autism spectrum disorder and co-occurring disruptive behavior. Biol. Psychiatry Cogn. Neurosci. Neuroimaging 4, 1031-1041. doi: 10.1016/j.bpsc. 2019.01.009

Jenkins, L. M., Andrewes, D. G., Nicholas, C. L., Drummond, K. J., Moffat, B. A., Phal, P., et al. (2014). Social cognition in patients following surgery to the prefrontal cortex. Psychiatry Res. 224, 192-203. doi: 10.1016/j.pscychresns. 2014.08.007

Jeon, H., and Lee, S.-H. (2018). From neurons to social beings: short review of the mirror neuron system research and its socio-psychological and psychiatric implications. Clin. Psychopharmacol. Neurosci. 16, 18-31. doi: 10.9758/cpn. 2018.16.1.18

Keltner, D., Sauter, D., Tracy, J., and Cowen, A. (2019). Emotional expression: advances in basic emotion theory. J. Nonverbal Behav. 43, 133-160. doi: 10.1007/s10919-019-00293-3

Kinoshita, M., Nakajima, R., Shinohara, H., Miyashita, K., Tanaka, S., Okita, H., et al. (2016). Chronic spatial working memory deficit associated with the superior longitudinal fasciculus: a study using voxel-based lesion-symptom mapping and intraoperative direct stimulation in right prefrontal glioma surgery. J. Neurosurg. 125, 1024-1032. doi: 10.3171/2015.10.JNS1591

Komatsu, S., Nakamura, T., and Hakoda, Y. (2012). Exprexxion Recognition Test for Adults. Hukuoka: Toyo Physical.

Kucharska-Pietura, K., Phillips, M. L., Gernand, W., and David, A. S. (2003). Perception of emotions from faces and voices following unilateral brain damage. Neuropsychologia 41, 1082-1090. doi: 10.1016/s0028-3932(02)00294-4

Lacoboni, M., and Dapretto, M. (2006). The mirror neuron system and the consequences of its dysfunction. Nat. Rev. Neurosci. 7, 942-951. doi: 10.1038/nrn2024

Li, Z., Santhanam, P., Coles, C. D., Ellen Lynch, M., Hamann, S., Peltier, S., et al. (2013). Prenatal cocaine exposure alters functional activation in the ventral prefrontal cortex and its structural connectivity with the amygdala. Psychiatry Res. 213, 47-55. doi: 10.1016/j.pscychresns.2012.12.005

Matsumoto, D., and Ekman, P. (1988). Japanese and Caucasian Facial Expressions of Emotion (JACFEE) and Neutral Faces (JACNeuF). San Francisco, CA: San Francisco State University.

Mattavelli, G., Pisoni, A., Casarotti, A., Comi, A., Sera, G., Riva, M., et al. (2019). Consequences of brain tumour resection on emotion recognition. J. Neuropsychol. 13, 1-21. doi: 10.1111/jnp.12130

Moor, B. G., Macks, Z. A., Guroglu, B., Rombouts, S. A., Molen, M. W., and Crone, E. A. (2012). Neurodevelopmental changes of reading the mind in the eyes. Soc. Cogn. Affect. Neurosci. 7, 44-52. doi: 10.1093/scan/nsr020

Morawetz, C., Bode, S., Baudewig, J., and Heekeren, H. R. (2017). Effective amygdala-prefrontal connectivity predicts individual differences in successful emotion regulation. Soc. Cogn. Affect. Neurosci. 12, 569-585. doi: 10.1093/scan/nsw169

Motomura, K., Terasawa, Y., Natsume, A., Iijima, K., Chalise, L., Sugiura, J., et al. (2019). Anterior insular cortex stimulation and its effects on emotion recognition. Brain Struct. Funct. 224, 2167-2181. doi: 10.1007/s00429-01901895-9

Nakada, M., Nakajima, R., Okita, H., Nakade, Y., Yuno, T., Tanaka, S., et al. (2020). Awake surgery for right frontal lobe glioma can preserve visuospatial cognition and spatial working memory. J. Neurooncol. doi: 10.1007/s11060-020-03656-9 [Epub ahead of print].

Nakajima, R., Kinoshita, M., and Nakada, M. (2020). Motor functional reorganization is triggered by tumor infiltration into the primary motor area and repeated surgery. Front. Hum. Neurosci. 14:327. doi: 10.3389/fnhum.2020. 00327

Nakajima, R., Kinoshita, M., Okita, H., Yahata, T., Matsui, M., and Nakada, M. (2018). Neural networks mediating high-level mentalizing in patients with right cerebral hemispheric gliomas. Front. Behav. Neurosci. 12:33. doi: 10.3389/fnbeh.2018.00033

Nakajima, R., Kinoshita, M., Okita, H., Yahata, T., and Nakada, M. (2019). Glioma surgery under awake condition can lead to good independence and functional outcome excluding deep sensation and visuospatial cognition. Neurooncol. Pract. 6, 354-363. doi: 10.1093/nop/npy054

Papagno, C., Pisoni, A., Mattavelli, G., Casarotti, A., Comi, A., Fumagalli, F., et al. (2016). Specific disgust processing in the left insula: new evidence from direct electrical stimulation. Neuropsychologia 84, 29-35. doi: 10.1016/j. neuropsychologia.2016.01.036

Perry, A., Saunders, S. N., Stiso, J., Dewar, C., Lubell, J., Meling, T. R., et al. (2017). Effects of prefrontal cortex damage on emotion understanding: EEG and behavioural evidence. Brain 140, 1086-1099. doi: 10.1093/brain/ awx031

Pertz, M., Okoniewski, A., Schlegel, U., and Thoma, P. (2020). Impairment of sociocognitive functions in patients with brain tumours. Neurosci. Biobehav. Rev. 108, 370-392. doi: 10.1016/j.neubiorev.2019.11.018

Phan, K. L., Wager, T., Taylor, S. F., and Liberzon, I. (2002). Functional neuroanatomy of emotion: a meta-analysis of emotion activation studies in PET and fMRI. NeuroImage 16, 331-348. doi: 10.1006/nimg. 2002.1087

Puglisi, G., Sciortino, T., Rossi, M., Leonetti, A., Fornia, L., Conti Nibali, M., et al. (2018). Preserving executive functions in nondominant frontal lobe glioma surgery: an intraoperative tool. J. Neurosurg. 131, 474-480. doi: 10.3171/2018. 4.JNS18393

Rizzolatti, G., Fadiga, L., Gallese, V., and Fogassi, L. (1996). Premotor cortex and the recognition of motor actions. Cogn. Brain Res. 3, 131-141. doi: 10.1016/0926-6410(95)00038-0

Rolland, A., Herbet, G., and Duffau, H. (2018). Awake surgery for gliomas within the right inferior parietal lobule: new insights into the functional connectivity gained from stimulation mapping and surgical implications. World Neurosurg. 112, e393-e406. doi: 10.1016/j.wneu.2018. 01.053

Saarimäki, H., Gotsopoulos, A., Jääskeläinen, I. P., Lampinen, J., Vuilleumier, P., Hari, R., et al. (2016). Discrete neural signatures of basic emotions. Cereb. Cortex 26, 2563-2573. doi: 10.1093/cercor/bhv086

Saito, T., Muragaki, Y., Maruyama, T., Tamura, M., Nitta, M., Tsuzuki, S., et al. (2016). Difficulty in identification of the frontal language area in patients with dominant frontal gliomas that involve the pars triangularis. J. Neurosurg. 125, 803-811. doi: 10.3171/2015.8. JNS151204

Shioiri, T., Someya, T., Helmeste, D., and Tang, S. W. (1999). Misinterpretation of facial expression: a cross-cultural study. Psychiatry Clin. Neurosci. 53, 45-50. doi: 10.1046/j.1440-1819.1999.00469.x

Southwell, D. G., Hervey-Jumper, S. L., Perry, D. W., and Berger, M. S. (2016). Intraoperative mapping during repeat awake craniotomy reveals the functional plasticity of adult cortex. J. Neurosurg. 124, 1460-1469. doi: 10.3171/2015.5. JNS142833

Suslow, T., Kugel, H., Rufer, M., Redlich, R., Dohm, K., Grotegerd, D., et al. (2016). Alexithymia is associated with attenuated automatic brain response to facial emotion in clinical depression. Prog. Neuropsychopharmacol. Biol. Psychiatry 65, 194-200. doi: 10.1016/j.pnpbp.2015.10.006

Suzuki, A., Hoshino, T., Shigemasu, K., and Kawamura, M. (2006). Disgustspecific impairment of facial expression recognition in Parkinson's disease. Brain 129, 707-717. doi: 10.1093/brain/awl011

Tate, M. C., Herbet, G., Moritz-Gasser, S., Tate, J. E., and Duffau, H. (2014). Probabilistic map of critical functional regions of the human cerebral cortex: broca's area revisited. Brain 137, 2773-2782. doi: 10.1093/brain/ awu168

Thiebaut de Schotten, M., Urbanski, M., Duffau, H., Volle, E., Levy, R., Dubois, B., et al. (2005). Direct evidence for a parietal-frontal pathway subserving spatial awareness in humans. Science 309, 2226-2228. doi: 10.1126/science.11 16251

Touroutoglou, A., Lindquist, K. A., Dickerson, B. C., and Barrett, L. F. (2015). Intrinsic connectivity in the human brain does not reveal networks for 'basic' emotions. Soc. Cogn. Affect. Neurosci. 10, 1257-1265. doi: 10.1093/scan/ nsv013

Tracy, J. L., and Randles, D. (2011). Four models of basic emotions: a review of ekman and cordaro, izard, levenson and panksepp and watt. Emot. Rev. 3 , 397-405. doi: 10.1177/1754073911410747

Uono, S., Sato, W., Kochiyama, T., Sawada, R., Kubota, Y., Yoshimura, S., et al. (2017). Neural substrates of the ability to recognize facial expressions: a voxel-based morphometry study. Soc. Cogn. Affect. Neurosci. 12, 487-495. doi: $10.1093 /$ scan/nsw142

Vytal, K., and Hamann, S. (2010). Neuroimaging support for discrete neural correlates of basic emotions: a voxel-based meta- 
analysis. J. Cogn. Neurosci. 22, 2864-2885. doi: 10.1162/jocn.2009. 21366

Yordanova, Y. N., Duffau, H., and Herbet, G. (2017). Neural pathways subserving face-based mentalizing. Brain Struct. Funct. 222, 3087-3105. doi: 10.1007/ s00429-017-1388-0

Yuvaraj, R., Murugappan, M., Norlinah, M. I., Sundaraj, K., and Khairiyah, M. (2013). Review of emotion recognition in stroke patients. Dement. Geriatr. Cogn. Disord. 36, 179-196. doi: 10.1159/000353440

Zhao, K., Zhao, J., Zhang, M., Cui, Q., and Fu, X. (2017). Neural responses to rapid facial expressions of fear and surprise. Front. Psychol. 8:761. doi: 10.3389/fpsyg. 2017.00761
Conflict of Interest: The authors declare that the research was conducted in the absence of any commercial or financial relationships that could be construed as a potential conflict of interest.

Copyright (c) 2021 Nakajima, Kinoshita, Okita, Liu and Nakada. This is an open-access article distributed under the terms of the Creative Commons Attribution License (CC BY). The use, distribution or reproduction in other forums is permitted, provided the original author(s) and the copyright owner(s) are credited and that the original publication in this journal is cited, in accordance with accepted academic practice. No use, distribution or reproduction is permitted which does not comply with these terms. 